\title{
IN VIVO STUDIES ON POSSIBLE HEALTH CONSEQUENCES OF GENETICALLY MODIFIED FOOD AND FEED-WITH PARTICULAR REGARD TO INGREDIENTS CONSISTING OF GENETICALLY MODIFIED PLANT MATERIALS
}

\author{
IAN F. PRYME* AND ROLF LEMBCKE**
}

*Department of Biochemistry and Molecular Biology, University of Bergen, Årstadveien 19, NO-5009 Bergen, Norway, and **Oplysning om Genteknologi, Hvidehusvej 5, DK-3450 Allerød, Denmark

\begin{abstract}
This synopsis reviews published in vivo studies on possible health consequences of genetically modified food and feed where the ingredients in question have consisted of genetically modified plant materials. The following, however, have not been taken into consideration:

-ingredients consisting of genetically modified microorganisms or parts of animals/fish -ingredients produced by/from genetically modified organisms but without any DNA present -studies on consequences for the environment or biodiversity

-in vitro studies or computer simulations

According to a Norwegian report "Gen-mat" (NOU 2000:29), and a more recent search in Medline and Citations Index, to our knowledge a total of ten studies have been published on the health effects of GM-foods and feeds. In this minireview the data made available in these published studies is discussed.

In their study Noteborn et al (1995) used a GM-tomato developed at a Dutch research institute, with a gene expressing the $\mathrm{Bt} \operatorname{Cry} 1 \mathrm{~A}(\mathrm{~b})$ toxin. The toxin was expressed at as little as $7,5 \mathrm{ng} / \mathrm{mg}$ protein in the fresh GM-tomato. A commercial semi-synthetic animal feed (no composition given), and the same feed mixed with $10 \%$ of lyophilised tomatoes (the GM-tomato or the unmodified parent) were fed to young male and female rats (weanlings) for 91 days. Rats given tomato-diets ate on average what corresponds to $20 \mathrm{~g}$ of fresh tomatoes a day. Percent survivals, terminal body weights and organ weights

*Corresponding author: Professor Ian F. Pryme, Department of Biochemistry and Molecular Biology, University of Bergen, Årstadveien 19, NO-5009 Bergen, Norway, tel. +47 55 586438, fax +4755586400, e-mail ian.pryme@ibmb.uib.no
\end{abstract}


(liver, kidneys, testes), and macroscopic examination of organs and tissues revealed no difference between GM-tomatoes and the unmodified parent. A histopathological investigation was referred to as "in progress" but has not yet been published. It would seem likely that the inclusion of $10 \%$ freeze-dried tomatoes distorted the diets' amino acid pattern. Moreover, at this inclusion level the Bt toxin (a GM tomato protein) intake of the rats was insignificant (the Bt expression level was one twentieth of that which is found in other Bt crops). Thus one could expect nothing significant to be found and this indeed was duly confirmed by the authors. It is evident that all acute toxicity and stability studies on Bt toxin were performed using an $E$. coli recombinant and not that isolated from the GM tomato. It needs to be emphasised that the structure and stability of the E. coli recombinant Bt toxin which they tested is almost certainly different from that synthesised by the plant. Indeed, in a later communication from the EU it was stated that the Bt toxin in the plant was glycosylated while neither $B$. thuringiensis nor $E$. coli would be able to perform this type of post-translational modification of the recombinant protein. An important observation was the fact that in vitro Bt toxin could bind to gut histology sections obtained from humans and rhesus monkeys. It must be pointed out that this is a book chapter and not a peer-reviewed paper.

Hammond et al (1996) utilised Monsanto's commercial RR soybeans, of the two lines 40-3-2 and 61-67-1, both with a gene that makes them RoundUp tolerant. A commercial rodent diet (with indicated chemical profile) and the same diet reformulated to contain $25 \%$ soy meat (from either of the two GMlines, or the unmodified parent) were fed to male and female rats eight weeks of age for one month. The GM and parental line soybeans were grown from the same field test sites. The soybeans were all processed (dehulled, defatted, toasted) and ground to meal. The feed contained $25 \%$ protein. Terminal body weights and relative organ weights (liver, kidneys, testes) did not show significant differences (no data given). There were no gross pathologic findings related to genetic modification (no information about which organs were examined). From the (rather poor) bar diagrams in the paper there was a significant difference in the growth rate of rats and catfish with the two different GM lines, even at the dietary protein levels which were artificially too high (close to 25\%). This very high protein content of the diet would almost certainly mask, or at least effectively reduce, any possible effect of the transgene, particularly when the inclusion level of the GM soya in any case was low. It is therefore highly likely that all GM effects would have been diluted out. No data were given for most of the parameters but the authors give a bland assurance that there were no differences. In the unprocessed soy study the inclusion level of the GM soy was too low and would probably ensure that any possible undesirable GM effects did not occur.

Additionally, a similar study was performed with unprocessed beans. Furthermore, chickens, catfish and dairy cattle were fed similar diets in order to study the feeding values of the two lines of GM-soybeans relative to the parental cultivar. 
In a SOAFED flexible fund project, coordinated by Pusztai (1998), a study was performed using two GM-potato lines developed at a Scottish Research Institute, both expressing the lectin GNA from snowdrop (Galanthus nivalis), which makes them resistant to insects and nematodes (but is supposed not to be toxic to humans). There were four feeding groups in each experimental series. All groups (consisting of male 19 day-old rats) were fed a diet (fully specified) for 10 or 110 days. In each the feed contained potatoes $(65,70$ or $76 \%$ on a dry weight basis), either from one of the two GM-lines, or from the parental line (grown from the same field test site as the GM-line), or from the parental line spiked with free GNA. Additionally there were controls formulated similarly but without potatoes, and there were controls with a standard rat feed. The diets in the four series were formulated to different protein contents $(6 \%, 8-9 \%$ or $15 \%$ ). Terminal body weights and organ weights (heart, lungs, testes, liver, kidneys, thymus, prostate, spleen, pancreas, jejunum, caecum, colon, soleus and gastrocnemius) were studied. It is evident from the results that differences in these weights related to differences in diets can only be ascertained using detailed statistical analyses of primary data. Measurements on the mucosa of the stomach lining and crypt lengths at various points in the intestine have been reported by Ewen and Pusztai (1999). In a recent follow-up gut histology study Pusztai (2002) has reported indications of a trophic effect caused by an unknown growth or proliferative signal present in the GM potatoes, since a hyperplastic growth of the small intestine was observed in rats fed GM potato. Interestingly the jejunum was not enlarged when the parent line diet was supplemented with GNA, the gene product. This observation supported previous results which had clearly shown that GNA, even when included in the diet at a several hundred-fold concentration compared with that expressed in the GM potato lines, had only a minor growth stimulatory effect on the jejunum (Pusztai et al., 1990).

Brake and Vlachos (1998) used a hybrid of Novartis' Event 176 GM-corn, expressing the Bt Cry1A(b) toxin. A diet (fully specified) with $61 \%$ (later 67\%) corn (from either the GM-line, or the unmodified parental line, which was grown, processed and stored concurrently with the GM-line) was fed to male and female one day old broiler chickens for 38 days. The protein contents of the diets were $250 \mathrm{~g} / \mathrm{kg}$ dry weight (later $230 \mathrm{~g} / \mathrm{kg}$ ). No differences were reported neither in percent survivals of the chickens nor in their body weights (determined as a total for each pen, containing 40 chickens). No differences were reported between chickens fed diets in mashed or in pelleted form (the latter involving a treatment that degrades proteins). All birds in every treatment were combined and thus no individual data are available. Additionally, feed conversion ratios, and carcass and parts yields were investigated. No histological examinations are reported. It must be pointed out that this was a commercial, and not a scientific study.

In their study Fares and El-Sayed (1998) used GM-potatoes developed at an Egyptian institute, with a gene expressing the Bt Cry1A(b) $\delta$-endotoxin. Potatoes (presumably raw, whole potatoes as the sole ingredient of the diets) 
were fed to three groups of one month old mice for two weeks. Each group was fed either GM-potatoes, or (unspecified) conventional potatoes, or the same treated with free $\delta$-endotoxin. The ileum was studied in detail by both light and electron microscopy. Changes were observed in the structural configuration of the ileum of mice fed on GM potatoes, and even more serious defects were observed in mice fed $\delta$-endotoxin treated potatoes.

Ewen and Pusztai (1999) studied one of the GM-potato lines developed in the SOAFED flexible fund project (Pusztai, 1998). In this histopathological study a fully specified diet containing $76 \%$ of boiled or raw potatoes (from either the GM line, or the parental line, or the latter with free GNA added) was fed to male rats nineteen days of age for ten days. The diet contained $6 \%$ protein. Histological samples of the mucosal linings of stomach, jejunum, ileum, caecum, and colon were prepared and mucosal thickness (stomach) or crypt length (intestine) measured by video-image analysis. The mucosal thickness of the stomach was greater after feeding diets containing GNA (irrespective of whether GNA was added to parental line potatoes, or GNA was expressed in GM-potatoes) than after diets without GNA. On the other hand, crypt lengths in the jejunum of the rats fed on GM-potato diets were significantly greater than in those given parent-line potatoes (with or without added GNA). A significantly greater number of interepithelial lymphocytes was found after diets on GM-potato (raw or cooked), compared to diets on parental-line potatoes (raw or cooked).

In the study by Pusztai et al. (1999) a GM-pea developed at a US research institute was used. The GM pea contained a gene expressing kidney bean $\alpha$ amylase inhibitor. Fully specified diets with $15 \%$ protein was fed to nineteen day old male rats for ten days. The diets were made up with $30 \%$ or $65 \%$ peas (of either the GM or the parental line) or with no peas at all (a diet on a lactalbumin basis), the latter with or without pure bean $\alpha$-amylase inhibitor added. The GM and parental line peas were grown together in the same greenhouse. Final body weights, organ weights and body Nitrogen contents in rats given transgenic or parental line peas were not significantly different. Neither did analyses of caecum tissue (for DNA, RNA and protein), pancreas (for DNA, RNA, protein, trypsinogen, chymotrypsinogen and $\alpha$-amylase) and small intestinal lumen (for trypsin, chymotrypsin, $\alpha$-amylase, protein, and starch) reveal much difference. Interestingly, the very strong effect of the $\alpha$ amylase inhibitor when added to the lactalbumin diet, was all but absent when the inhibitor was expressed in the GM-peas. At 30\% dietary inclusion both caeca and pancreas were significantly different and some nutritional performance data were also different. At $65 \%$ the organ weight differences disappeared because the non-GM peas also contained a number of agents which increased the size of sensitive tissues (caecum, pancreas etc.) and may therefore have obliterated differences between GM and non-GM material. A drawback in this study is that no histological examinations are reported.

Hashimoto et al. (1999) utilised two GM-potatoes developed at a Japanese research institute, both expressing a glycinin, one a native glycinin, the other a 
designed glycinin with four methionyl residues in the molecule. A commercial diet (no composition given) was fed to four groups of male rats four weeks of age for 28 days. Three of the groups were at the same time fed homogenized potatoes (either from one of the two GM-lines, or from the parental line), using a metallic gastric tube. In this way, about 0.16 gram of the potatoes were given each rat daily (double the dose recommended by the Japanese guidelines for the assessment of food additives). Body weights, food intake, various haematological data, chemical blood data, organ weights and histopathological findings are reported The results indicate no real difference between the GMpotato lines and the parental line. It is apparent, however, that the glycinin expression level and its intake by the rats was far too low to make the studies of major interest.

In their study Teshima et al. (2000) used a genetically modified, glyphosatetolerant soybean line and a closely related and one-parent-same-cultivar line, produced in Ohio, U.S.A. and purchased from a Japanese trading corporation. The soybeans are stated to be commercially available and are, supposedly, from a line of Monsanto's RoundUp Ready soybeans. The statement about the nonGM soybeans is rather vague. Female B10A mice and female Brown Norwegian rats, both at seven weeks of age were fed, separately, in three groups, one with a diet (fully specified) containing 30\% GM soybeans and 7\% casein (in total $17-18 \%$ protein), another with a similar diet containing the unmodified soybeans, and a control group with a standard commercial rodent feed (CRF-1). The soybeans used were heated in an autoclave at $100^{\circ} \mathrm{C}$ for 30 minutes and ground. The three groups were fed their respective diets for 15 weeks. No significant differences were found for the accumulated body weights and the weights of liver and spleen between the three groups of animals. But it is remarkable that neither rats nor mice grew to any major extent in 15 weeks' time on this rather protein rich diet. Sera from the animals fed GM or non-GM beans were tested using an indirect ELISA. The results recorded are rather difficult to interpret, but are stated to indicate that "production of soybean-specific IgE was not detected in the sera of either group, and the increase in soybean-specific IgG was the same in both GM and non-GM groups". A thorough histopathological examination was performed. Thin stained sections of the liver were examined. Thymus, spleen, mesenteric lymph node, and Peyer's patches were inspected to evaluate the composition and arrangement of the cells. The structure of the crypt and the composition and forms of the mucosa in the small intestine were studied. No differences between the animals fed GM and non-GM soybeans were detected.

\section{COMMENTS}

Although very many have voiced their opinions both in the popular and scientific press there is only very limited data published in peer reviewed journals concerning the safety of GM food (Domingo, 2000). It would seem 
apparent that GM food regulation is currently based on a series of extremely insufficient guidelines.

This minireview shows that potatoes, peas and corn (maize) have been fed to test animals in feed containing up to $60-75 \%$ of these ingredients in order to test transgenic plant materials against their conventional counterparts. Hopefully, no food or feed additive is so toxic as to give adverse effects with the small dose used by Hashimoto et al. (1999).

Obviously there are difficulties in formulating the corresponding transgenic and nontransgenic diets so that they are both isocaloric and identical with respect to all measured components, when a large part of the feed must be made up of test material. Nevertheless, this was attempted in many of the studies, viz. Hammond et al., 1996; Pusztai 1998; Brake and Vlachos, 1998; Ewen and Pusztai 1999; Pusztai et al., 1999, Pusztai 2002. The feeding periods were 1014 days (Pusztai 1998; Fares and El-Sayed 1998, Ewen and Pusztai 1999; Pusztai et al., 1999; Pusztai 2002), 28-38 days (Hammond et al., 1996; Fares and El-Sayed 1998, Hashimoto et al., 1999) and 91-150 days (Noteborn et al., 1995; Pusztai 1998; Teshima et al., 2000).

It is generally recognised that samples of commercial GM plant materials are not obtainable without prior contractual understanding with the producer (Lembcke 2000). For this reason, only institutions able to develop and cultivate GM plants, themselves, have been regarded here as independent.

The work in five studies (Noteborn et al., 1995; Hammond et al., 1996, Brake and Vlachos, 1998; Hashimoto et al., 1999, Teshima et al., 2000) was regarded as having been performed more or less in collaboration with private companies. In none of these studies were effects related to GM-materials reported. On the other hand, adverse effects were reported (but not explained) in independent studies by Pusztai (1998, 2002), Fares and El-Sayed (1998), Ewen and Pusztai (1999) and Pusztai et al., (1999). It is remarkable that these effects have all been observed after feeding for only 10-14 days.

Pusztai's studies (Pusztai 1998, 2002; Ewen and Pusztai 1999; Pusztai et al., 1999) are remarkable in that the experimental conditions were varied and several ways were found by which to demonstrate possible health effects of GM-foods. Importantly, young rats were used throughout (during development the organism is highly dependent on appropriate feed) and low protein feed was tried (the idea being that health defects would show up earlier at low protein intakes). Potatoes with pure GNA added were compared to GM-potatoes expressing GNA (defects occurring with GM-potatoes and not with potatoes to which GNA had been added would likely be caused by the genetic modification process itself). Fares and El-Fayed (1998) used the same approach with Bt $\delta$ endotoxin, and Pusztai et al., (1999) proceeded similarly with bean $\alpha$-amylase inhibitor.

It has been suggested that GM-plant material should not only be compared with material from the parent plant, but also with material from the parent plant genetically modified to express the "empty construct", i.e. a construct (vector) without the transgene but otherwise similar to the construct used for the GM- 
plant (any effect observed with material from the plant with the empty construct, and not with material from the parent is likely to be caused by the genetic modification process itself). This has not yet been tested.

Close electron microscopic observation of intestine tissues, particularly of the ileum, chemical analysis of intestinal tissue and luminal contents of the intestines, as well as immunological studies with provocation by lectins such as ConA and PHA have produced results which deserve further study (Pusztai 1998, 2002; Fares and El-Fayed 1998; Ewen and Pusztai 1999; Pusztai et al, 1999).

In conclusion we feel that much more scientific effort and investigation is necessary before we can be satisfied that eating foods containing GM material in the long term is not likely to provoke any form of health problems. It will be essential to adequately test in a transparent manner each individual GM product before its introduction into the market.

\section{REFERENCES}

Brake, J. and Vlachos D. (1998). Evaluation of transgenic event $176 \mathrm{Bt}$ corn in broiler chicken. Poultry Sci. 77, 648-653.

Domingo, J.L. (2000). Health risks of genetically modified foods: Many opinions but few data. Science, 288, 1748-1749.

Ewen, S.W.B. and Pusztai, A. (1999). Effects of diets containing genetically modified potatoes expressing Galanthus nivalis lectin on rat small intestine. The Lancet, 354, 1353-1354. (described in more detail in Lembcke, 2000).

Fares, N.H. and El-Sayed, A.K. (1998). Fine structural changes in the ileum of mice fed on endotoxin-treated potatoes and transgenic potatoes. Natural Toxins, 6, 219-233.

Hammond, B.G., Vicini, J.L., Hartnell, G.F., Naylor, M.W., Knight, C.D., Robinson, E.H., Fuchs, R.L. and Padgette, SR. (1996). The feeding value of soybeans fed to rats, chickens, catfish and dairy cattle is not altered by genetic incorporation of glyphosate tolerance. $J$. Nutr. 126, 717-727.

Hashimoto, W., Momma, K., Yoon, H.J., Ozawa, S., Ohkawa, Y., Ishige, T., Kito, M., Utsumi, S. and Murata, K. (1999). Safety assessment of transgenic potatoes with soybean glycinin by feeding studies in rats. Biosci. Biotechnol. Biochem. 63, 1942-1946.

Lembcke, R. (2000). Dr. Pusztai's fors øg med gen-kartofler-døm selv. ISBN 87-987905-0-1 (in Danish).

Noteborn, H.P.J.M., Bienenmann-Ploum, M.E., van den Berg, J.H.J., Alink, G.M., Zolla, L., A. Reynerts, Pensa, M. and Kuiper, H.A. (1995). Safety assessment of the Bacillus thuringiensis insecticidal Crystal Protein CRY1A(b) expressed in transgenic tomatoes. In: Genetically modified foods. Safety issues. Eds.: K.-H. Engel, G.R. Takeola \& R. Teranishi. ACS Symposium Series 605, Washington DC, pp. 134-147.

Pusztai, A. (1998). SOAFED flexible fund project RO818 (a government funded project, coordinated by Dr Arpad Pusztai, involving three Scottish research institutes and described in detail in a recent Danish report (Lembcke 2000). Audit report of data produced at the Rowett Research Institute (RRI) (21st, August 1998). Copies of the reports were circulated to a number of well-known international scientists prior to the announcement of the memorandum (signed by 23 scientists) in defence of Dr. Pusztai and his team at the RRI. The reports were made public on the internet site of the RRI under the address http:// www.rri.sari.ac.uk/. In a later revision of the site these were withdrawn. This review is based on the circulated copies. 
Pusztai, A. (2002). Can science give us the tools for recognizing possible health risks of GM food'? Nutr. Health, 16, 73-84.

Pusztai, A., Ewen, S.W.B., Grant, G., Peumans, W.J., van Damme, E.J.M., Rubio, L. and Bardocz, S. (1990). Relationship between survival and binding of plant lectins during small intestinal passage and their effectiveness as growth factors. Digestion, 46 (suppl. 2), 308316.

Pusztai, A., Grant, G., Bardocz, S., Alonso, R., Chrispeels, M.J., Schroeder, H,E., Tabe, L.M. and Higgins, T.J.V. (1999). Expression of the insecticidal bean $\alpha$-amylase inhibitor transgene has minimal detrimental effect on the nutritional value of peas fed to rats at $30 \%$ of the diet. J. Nutr. 129, 1567-1603.

Teshima, R., Akiyama, H., Okunuki, H., Sakushima, J.-I., Goda, Y., Onodera, H, Sawada, J.-I. and Toyoda, M. (2000). Effect of GM and non-GM soybeans on the immune system of BN rats and B10a mice. J. Food Hyg. Soc. Japan, 41,188-193.

(Received 9 October 2002) 